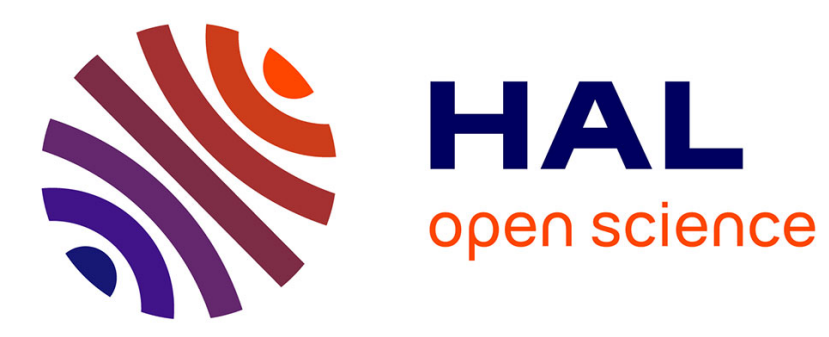

\title{
Emplacement et déplacement des écoles en milieu Kanak
}

Eddie Wadrawane

\section{- To cite this version:}

Eddie Wadrawane. Emplacement et déplacement des écoles en milieu Kanak. Les Sciences de l'éducation pour l'ère nouvelle: revue internationale, 2008. hal-03268165

\section{HAL Id: hal-03268165 \\ https://hal-unc.archives-ouvertes.fr/hal-03268165}

Submitted on 23 Jun 2021

HAL is a multi-disciplinary open access archive for the deposit and dissemination of scientific research documents, whether they are published or not. The documents may come from teaching and research institutions in France or abroad, or from public or private research centers.
L'archive ouverte pluridisciplinaire HAL, est destinée au dépôt et à la diffusion de documents scientifiques de niveau recherche, publiés ou non, émanant des établissements d'enseignement et de recherche français ou étrangers, des laboratoires publics ou privés. 


\section{Emplacement et déplacement des écoles en milieu Kanak}

Un analyseur anthropologique de la place faite aux institutions de diffusion du savoir occidental dans une situation coloniale Eddy Wayuone WADRAWANE*

Résumé : En Nouvelle-Calédonie et en milieu kanak c'est-à-dire dans ce qu'on appelle les tribus, l'école est dans la plupart des cas implantée loin de l'espace où se concentre la vie sociale du groupe. Hors de cet espace social/domestique, une frange périphérique lui est dévolue. Bien que singulièrement excentrée, l'école n'est ni intégrée, ni rejetée, mais placée à la position qui convient au pouvoir qui lui est accordé, juste là où il faut. L'histoire et l'actualité de ce mode d'implantation constituent un indicateur de la place accordée, par les Mélanésiens, aux institutions de diffusion du savoir occidental et à la place de ce savoir lui-même.

Mots-clés : École indigène. Espace Kanak. Rapport au Savoir. Nouvelle-Calédonie. Privince des Îles loyautés.

* Formateur chargé d'enseignement en Sciences de l'éducation et en langues et Culture Kanak, IUFM du Pacifique Nouvelle-Calédonie, Doctorant du laboratoire DAESL (Didactique et Anthropologie des Enseignements Scientifiques et Langagiers), Université Victor Segalen bordeaux 2. 
Les Sciences de l'éducation - Pour l'Ère nouvelle, vol. 41, $\mathrm{n}^{\circ} 1,2008$

«L'école, [...] avant quand on a accepté, on ne savait pas trop faire quoi avec cette chose. Mais on a dit, peut-être que c'est bon pour les gosses plus tard. Voilà pourquoi on a donné un bout de terrain pour l'école [...]».

Mayaet Salomon, petit chef de la tribu de Werap, Yehen (Hienhgen en Français, village de la côte Est).

«Pour savoir ce qu'ils font [les parents qui font baptiser leur enfant], je n’ai pas à interpréter le sens de ce qu'ils font à partir de leurs actions ou de leurs propos. Mieux vaudrait dire : ils agissent dans un monde où certaines actions sont sensées (admises, reconnues, plausibles, attendues...) et d'autres non. [...] Dans la mesure où leur action est conforme à des règles, elle peut être décrite.»

J. Bazin, 1998

\section{Introduction}

Une tradition anthropologique et sociologique a pris l'habitude, peut-être pour se dédouaner d'une mauvaise conscience post-colonialiste, d'étudier les relations entre l'institution scolaire et les peuples colonisés (qu'ils le soient encore, qu'ils soient en voie de décolonisation ou qu'ils aient acquis leur indépendance) sous l'angle de l'imposition brutale d'un modèle occidental et celui de la violence symbolique déqualifiant les modes culturels locaux. Comme l'écrit N. Wachtel dans l'introduction de son remarquable ouvrage La vision des vaincus: «les études d'acculturation portent essentiellement sur des sociétés de force inégale, l'une dominante, l'autre dominée; le terme se charge donc d'une hypothèque historique de suprématie et risque de traîner avec soi la vieille grille européo-centriste ${ }^{1} »$. Sans avoir la prétention de rétablir de manière exhaustive «la vision des vaincus» - qui ne le sont plus, $\mathrm{du}$ moins officiellement - nous prendrons ici le parti, en nous appuyant sur des données spatiales et temporelles objectives, de voir comment l'école, objet nouveau parmi d'autres objets nouveaux, étranger et étrange, a été considérée par les acteurs de communautés qui, à quelques exceptions près [Maré], ne pouvaient la comparer à quelque institution préexistante. Le fil rouge de notre contribution sera, comme

1. Wachtel N. La vision des vaincus. Paris : Gallimard, 1971, p. 24. 
l'indique le titre, l'étude systématique de la place faite par les «vaincus» au bâti scolaire dans l'espace des tribus.

Introduite d'abord par les missionnaires puis par l'administration, l'école fut de manière quasi continue, avec des accès de fièvres notoires, au centre de discussions et polémiques explicites ou non avouées. Nous n'entrerons pas ici dans une perspective polémique régulièrement alimentée de prises de positions politiques et idéologiques dépourvues de fondements anthropologiques solides. À l'heure où, pour tenter de trouver une énième solution à l'échec massif des jeunes issus précisément et des tribus et de la périphérie de Nouméa, on parle d'adaptation des programmes et de la place de la culture, notre proposition de nature anthropo-historique sera modestement un début d'analyse de la position spatiale paradoxale des écoles en milieu kanak. Après avoir posé quelques bases épistémologiques, nous tenterons de répondre aux questions suivantes, constitutives des différentes parties qui seront traitées dans cet article.

a. Qu'appelle-t-on et comment a-t-on construit la notion de "Centre de la tribu»? b. Comment ces écoles sont-elles agencées, quelles sont les caractéristiques de cet agencement et quelles contingences guident ces implantations singulières?

c. Paradoxalement entre «désir et/ou imposition d'école» quelles sont les stratégies adoptées par les Kanak?

Après une brève synthèse conclusive nous déclinerons la direction ou l'orientation dans laquelle suivra le fil de nos travaux futurs.

\section{Sociologie, Sociohistoire et Anthropologie}

En 1774, Cook capitaine de la marine britannique, aborde sur une île qui sera 'baptisée' Nouvelle-Calédonie. À partir de 1830, les contacts entre Kanak et Occidentaux se multiplient. Le 24 septembre 1853, le contre amiral Fébvrier Despointes, prend possession de l'île au nom de la France impériale. Progressivement la société coloniale se met en place. Un embryon d'école missionnaire s'implante dès l'arrivée des religieux et l'installation avec son cortège de micro institutions. Des «écoles du dimanche» et des lieux de catéchisme sont créés. Chez les protestants, la bible devient l'instrument principal d'enseignement de la lecture et de l'écriture auprès des enfants de tribus. Cependant comme le souligne Pierre Clanché2 :

2. CLANCHÉ P. Histoire d'implantation et d'investissement des lieux d'instruction, et représentation de la transmission des savoirs. In : PEYRONIE H. \& VERGNIOUX A. (dir.). Éducation et longue durée. Caen : PUC, 2007, p. 107. 
"Ce n'est que bien plus tard que certaines écoles privées catholiques et surtout protestantes deviendront quasi officiellement les "écoles des Kanak" reconnaissant d'abord la dignité de leur culture, telle celle fondée par le Pasteur Leenhardt à Houailou, le mythique collège Do Néva, qui avant de devenir le point de passage obligé de générations de jeunes Kanak, n'était à l'origine (1904) qu'une petite école pastorale formant les futurs diacres ${ }^{3} »$.

Côté administratif par contre, il faut attendre les années 1880 pour pouvoir parler d'une esquisse de système scolaire indigène. En 1884, deux textes relatifs à la spécificité scolaire, statuent officiellement sur la mise en place et le développement des écoles indigènes. Le " 27 mai, un arrêté [prévoit] la création d'écoles dans les tribus de la Grande Terre et des îles Loyauté, complété le 11 juillet par un arrêté portant création de fonctions de moniteurs adjoints pour des écoles indigènes ${ }^{4}$ ». Symboliquement peut-être, les plus anciennes écoles ont été établies à Belep (île de l'extrême Nord) et à l'Ile des pins (extrême Sud). L'histoire qui s'en suivra est magistralement analysée par M. Pineau Salaün dans L'école indigène, Nouvelle-Calédonie 1885-1945, ouvrage issu d'une thèse soutenue en 1999 Les Kanak et l'école, Socio-histoire de la scolarisation des Mélanésiens de Nouvelle-Calédonie (1853-1998).

Dans les années 1970, suite au retour de métropole des quelques étudiants et militaires Kanak et Calédoniens ayant séjourné en France durant la période 68, la contestation du système scolaire accusé de colonial devient vive. Elle est relayée par quelques coopérants de la même génération venus pour faire leur service en tant que VAT et affectés dans les écoles de brousse et des îles de Loyauté. Le discours sur l'échec scolaire massif des Kanak devient un support prépondérant dans la contestation du système politique en général.

En 1985, J. M. Kohler et L. J. D. Wacquant publient sous l'égide de l'ORSTOM L'école inégale; Éléments pour une sociologie de l'école en Nouvelle Calédonie. Ils font une analyse très critique de l'école calédonienne. S'appuyant sur la théorie bourdieusienne de la reproduction (P. BOURDIEU, J.C. PASSERON, 1970), ces auteurs dénoncent le décalage entre le système scolaire et la classe «des minorités». L'école et les enseignants s'avèrent être les vecteurs inconscients de la reproduction des inégalités sociales et des discriminations. Pour eux «la question scolaire ne se résoudra qu'avec la fin de la domination bourgeoise [blanche] sur une communauté séculaire exploitée». À l'inverse et dans le même temps, M. J. Dardelin (1984) pense plutôt le problème scolaire en terme de rapport ethnique et culturel, le décalage étant au centre même des

3. Clifford J. Maurice Leenhardt, personne et mythe en Nouvelle-Calédonie. Paris : Jean Michel Place, 1987.

4. Arrêté du 15 octobre 1885 décrété par le gouverneur de Nouvelle-Calédonie portant règlement sur l'instruction publique. 
différences culturelles; son point de vue, taxé de culturalisme naïf est très violemment contesté par les marxistes purs et durs. Suite aux «évènements» - euphémisme communément partagé pour désigner les actions engageant une lutte ouverte pour l'indépendance - la création en 1985 des Écoles Populaires Kanak, comme alternative radicale à l'école coloniale, oriente alors le débat sur la pertinence des objets de connaissance dans l'école. J. Gauthier (1996) plaide pour la prise en compte des Écoles Populaires Kanak créées au lendemain des «événements» de 1984. Il tient un discours apologétique sur cette structure en interpellant plusieurs interlocuteurs locaux à l'appui de sa thèse. Cependant dans le milieu kanak les avis demeurent partagés sans doute par crainte d'enfermement ou d'incompréhension. Témoin la déclaration de M. Washetine ${ }^{5}$ qui pense "qu'il vaudrait mieux réfléchir à quelque chose à l'intérieur de l'institution, comme les langues et la culture» (communication personnelle, 2004). En outre un pragmatisme appliqué et ponctuel est insuffisant dans ce genre de projet. Finalement tous ces discours convergents, divergents et/ou oppositionnels ont sans doute dévié et dissimulé aux acteurs scolaires les véritables problématiques éducatives. Encore plus, maintenir et perpétuer une description globalisante du système scolaire est à notre sens insuffisant. Comme le disent les militants du Palika «Nous devons repenser ${ }^{6}$ autrement l'École ${ }^{7}$ ».

La recherche de type socio-anthropologique sur l'école englobant l'aspect historique a alors toute sa raison d'être. C'est ce que tente de faire M. Pineau Salaün en recourant aux principes de l'histoire orale auprès des anciens acteurs de l'école Kanak, en particulier des «moniteurs». Si aujourd'hui l'ignorance des enseignants locaux «de la vie réelle des enfants de tribus et des stratégies d'évitements développées» (CLANCHÉ, 1996) est manifeste, c'est qu'on s'est trop attardé à décrire cette école en regrettant un prétendu faible empan socioculturel mais surtout en la déconnectant de son contexte spatio-temporel. Dans cette perspective, il était intéressant de reconsidérer la situation spatiale de l'école dans la tribu.

L'école, en effet, s'insère dans un "contexte entrelacé» (BENSA, 1998) à l'intérieur duquel chaque élément peut à tout moment être redéfini en fonction des différents liens qui en tissent la trame. Le contexte au sens de Naepels «n'est pas le décor stable d'une pièce de théâtre, ce ne sont pas les règles établies pour toujours d'un jeu, ce n'est pas le cadre macroscopique de l'infinité des actions microscopiques

5. Michel Washetine est directeur de SEGPA à Bourail.

6. Cf. Les 30 ans du PALIKA, Poindimié, 2006.

7. Pour exemple, entre 2004 et 2006, les parents de l'île d'Ouvéa, Iaaï, ont manifesté ouvertement et publiquement leur désir devant l'Etat d'édifier un collège public sur l'île. Or, le secondaire est principalement pris en main par l'enseignement confessionnel. À cette époque j'étais le Directeur de l'Enseignement de la Province des îles Loyauté. 
Les Sciences de l'éducation - Pour l'Ère nouvelle, vol. 41, $\mathrm{n}^{\circ} 1,2008$

des hommes mais simplement l'ensemble de ce qui définit à un moment donné le possible et le légitime, et qui ne cesse de se transformer, serait-ce très lentement, au fil des actions, des coups de force, des oublis ou des intérêts changeants de chacun" (NAEPELS, 1998 : 18); il est un élément pertinent d'analyse de ce positionnement à propos de l'école. Ce contexte entrelacé permettrait de cerner les phénomènes de transferts stratégiques entre ce qui est «autour de l'école» et ce qui est à «l'intérieur de l'école» (WOODS, 1990), autrement dit un ensemble de phénomènes liés au «croisement entre le contrat didactique et le contrat coutumier ${ }^{8}$ » (ClanCHÉ, 2000).

Nous pensons cependant que cette étude suppose un préalable : l'examen à la fois de ce qui se passe «autour de l'école» et de sa position spatiale dans la tribu. Car par hypothèse, un statut spécifique aux structures institutionnelles de savoir occidental y est induit. Nous limitons ponctuellement nos propos à l'analyse de la position spatiale de l'école dans la tribu.

\section{Les tribus ont-elles un centre?}

La tribu, institution récente d'appellation exogène directement liée à la sédentarisation, présente une structure géospatiale particulière qui la distingue du village ou du bourg. Sans entrer dans des considérations géographiques et démographiques pour lesquelles nous n'avons pas de compétence, on peut dire que les tribus ont une structure unilinéaire plus ou moins sinusoïdale comportant quelques embranchements se terminant en cul de sac, à la différence de la structure en croix ou radiale des bourgs ou petites villes de métropole. La longueur de la sinusoïde est, aux yeux de l'observateur étranger, disproportionnée par rapport à la faible densité de population $( \pm 200$ habitants répartis sur $\pm 4 \mathrm{~km}$ de chemin principal et petits embranchements). Pour dire les choses un peu crûment la civilisation Kanak n'est pas une civilisation urbaine ou qui tendrait à le devenir. Historiquement les Kanak sont des agriculteurs et/ou pécheurs et le restent dans leur mode d'occupation de l'espace. Donc la tribu est une sorte de long fil avec une légère densification en son milieu autour et de part et d'autre de certains bâtiments.

Ce que l'on peut appeler le «Centre» de la tribu, bien que ce terme ne soit jamais utilisé, est identifiable par l'édifice religieux où s'organisent les nouveaux lieux de

8. Clanché P. Anthropologie de l'éducation des mathématiques : pour une anthropo-didactique. In : DAEST, Actes de Colloque, Marseille, 2000, 16 p. 
culte, Huhnami, ou Pahnameneng'. Les populations sont regroupées autour des églises pour former ces lieux de cultes devenus des tribus (SAND, BOLE, OUETCHO, 2000). Deux autres bâtiments/espaces publics sont généralement implantés à proximité du lieu de culte : la maison commune et l'espace de jeux/sports. Par commodité nous pouvons schématiser une aire triangulaire virtuelle dont les angles sont l'église ou le temple, la maison commune (lieu de réunions) et le terrain de sport collectif (volley, pétanque, cricket...). Il est possible alors de déterminer un point « $\mathrm{B}$ » barycentre de ce triangle que nous considérerons ici comme le centre virtuel des activités sociales et domestiques. C'est à partir de ce point que nous mesurerons la position spatiale de l'école. De ce centre nous proposons d'étudier le positionnement de l'école.

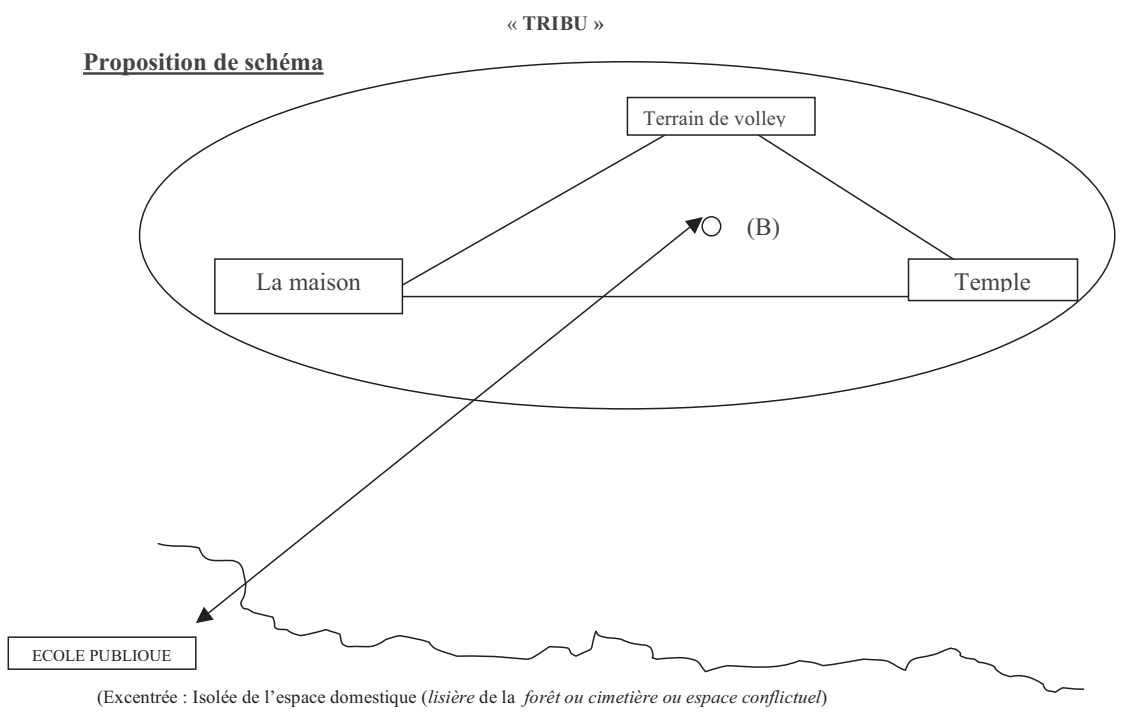

Les nombreux positionnements que nous avons examinés montrent clairement que l'école est non seulement décentrée, éloignée du centre virtuel ainsi que des autres lieux domestiques, mais qu'elle est excentrée dans une frange périphérique et singulière qui lui est dévolue. Bref, elle n'est pas dans la tribu (WADraWANe, 2001). Doumenge

9. Le centre religieux est aussi choisi du fait de la présence du EIKA (ACRE : God's little ACRE). Terme anglo-saxon déformé (prononciation) qui signifie «le petit arpent du bon Dieu», lieu d'activité sociale intense dans la tribu. (D’après Shekketon). Huhnahmi $<H u$ «lieu» + hna $+h m i>$ " prier», Pahnameneng $<$ Pah «devant»+ (h) na+meneng> (asseoir ou assis ou maison). Ces termes désignent «la tribu» en langue Nengone et Drehu ce qui se traduit par «Lieu de culte». 
Les Sciences de l'éducation - Pour l'Ère nouvelle, vol. 41, $\mathrm{n}^{\circ} 1,2008$

le signalait déjà en 1982 «L'école et le dispensaire sont immanquablement rejetés aux extrémités du village», (DOUMENGE, 1982). Nous pensons que cet agencement géographique mérite d'être étudié à la lumière d'un certain nombre d'arrière-plans historicopolitico-culturels locaux et qu'il n'est pas sans relation avec «la perception du savoir occidental en milieu kanak ${ }^{10}$ ».

\section{Un positionnement complexe}

\subsection{L'école singulièrement excentrée}

L'insertion spatiale de l'objet nouveau est loin d'être anodine ou innocente. L'école ne s'enracine pas n'importe comment dans l'espace. En effet, les structures scolaires sont érigées sur des parcelles attribuées par les propriétaires fonciers et, si leur installation proprement dite (construction, clôture) ne pose apparemment pas de difficultés majeures, on est toutefois bien loin de soupçonner la complexité des raisons sociales qui président à son implantation. Les véritables raisons de ces localisations ne sont perçues que par ceux qui manipulent ces nouveaux objets en fonction d'intérêts politico-économiques, symboliques et religieux.

De même que l'étranger est accueilli avec courtoisie et curiosité dans la tribu pour peu qu'il se donne la peine de se présenter avec un peu d'humilité - pour dire vite, pour peu qu'il fasse une "petite coutume» - de même on sait aussi que les Mélanésiens ne dédaignent pas de voir des objets nouveaux s'implanter dans leur milieu familier : d'abord les terrains de cricket surtout dans les îles Loyauté, puis les terrains de football marqués par les poteaux de but, les filets du terrain de volley-ball d'abord en terre battue, maintenant sur une dalle de béton et dernièrement les terrains de pétanques pour les «vieux». Cependant, quand il s'agit de désigner leur place dans l'espace, les intéressés font appel à des critères socioculturels sans rapports dans un premier temps avec les nécessités éducatives telles que les concepteurs de l'école les envisagent. Il fallait, dès le début de l'implantation, user de stratégie. Consentir à la volonté du pouvoir étranger mais aussi chercher à s'approprier l'objet imposé, ne serait-ce que dans sa forme. Le monde mélanésien décide du statut appliqué à tout élément, nouveau ou pas, qui intègre son espace de vie. L'école est ainsi... singulièrement... excentrée.

10. Cf. notre thèse en cours d'écriture : «Situation spatiale paradoxale de l'école en milieu kanak : Rapport aux institutions de diffusion du savoir occidental dans une situation coloniale (titre indicatif)». 


\subsection{L'école posée «dans» un tel lieu... Stratégie et consensus}

Tout comme l'igname, symbole de richesse, toujours "posée” à terre lors de sa présentation aux dignitaires claniques, l'école l'est aussi "dans" 11 ou sur un tel lieu. Elle n'est pas rejetée mais "posée"12. Il n'est pas rare de voir s'organiser un espace horticole autour des écoles, surtout pour les plus anciennes. Dans ce jardin aménagé de l'école, certaines variétés florales importées peuvent se mêler à l'igname, la canne à sucre, le bananier et le taro traditionnel.

Sur l'île de Maré, l'école de Nécé (district de Guahama), était installée à ses débuts sur le terrain clanique des Acania-doku, (Atressi à Lifou, littéralement «les maîtres du mal», traduction proposée par C. Illouz, 2000). Nous préférons garder les termes vernaculaires Acania-doku et Atressi afin d'éviter les ambiguïtés liées à la traduction. Tout d'abord située à l'intérieur des terres, l'école fut ramenée par la suite sur le littoral. L'intérêt n'était pas tant sa situation à proximité de la mer que le fait d'être agencée dans l'espace de ceux qui s'occupent des affaires internes de la chefferie. La première école de Tadine obéit au même principe d'installation. Ceci est confirmé par Welepa Welepa, membre du clan Acania, ancien élève de l'école et qui résida au lieu même où fut implantée la première infrastructure. La même disposition s'applique à l'école de Mou à Lifou, appréhendée également comme un objet nouveau et convoité. Il n'est donc pas étonnant qu'elle passe d'abord par cette phase qu'on peut qualifier d'“accueil stratégique". La position spatiale de l'infrastructure scolaire illustre cet accueil.

Outre son accueil stratégique, l'école, comme objet nouvellement introduit, bénéficie du statut d'objet consensuel. Bien souvent, l'école publique assume ce rôle lorsque deux positions litigieuses, religieuses ou claniques, ne sont pas parvenues à un accord mutuel; une zone limitrophe entre ces deux influences est alors négociée. Les écoles de Dozipà Lifou, de Netchaot au centre nord de la Grande Terre et de Tadine à Maré, témoignent de ce compromis.

L'école publique de Tadine offre encore un bel exemple de ce type. Comme le souligne Welepa : «L'école de Tadine est une des premières de Maré. [...]. Le Grand Chef a demandé aux clans $\mathrm{X}$ et $\mathrm{Y}^{13}$, le terrain [...] eux non plus ils n'arrêtent pas de se bagarrer pour ce terrain [...] En mettant l'école là, ça devient un lieu public. Ils ont fait la coutume car la mairie et l'école chevauchent le terrain de plusieurs propriétaires fonciers. [...] En fait, ce sont des problèmes de limites. Depuis il n'y a plus de

11. Il nous paraît judicieux d'utiliser le concept «dans» car l'école intègre un espace contextuel qui est bien plus que géographique (c'est nous qui soulignons).

12. Lors des cérémonies coutumières, tous les objets donnés - les coutumes - sont toujours posés à terre avec précautions devant les personnes qui vont "parler sur... ou à propos de...».

13. Lettres substituées aux véritables noms des personnes pour leur garantir l’anonymat. 
problème, parce que les limites litigieuses sont couvertes par [...] l'école et la mairie [...]. Lélément étranger sert de base de réconciliation entre les clans quand il y a des différends» (WeLEPA, 2000. Communication personnelle). Cet espace public surgit d'une manière consensuelle dans le milieu et constitue pour ainsi dire une création juridique et non un lieu imposé arbitrairement par l'extérieur.

Examinons maintenant le cas de l'école de la tribu de Netchaot (Grande Terre, Province Nord). D'après nos informateurs de Netchaot rencontrés chez le pasteur, Oudare N., Kowy (clan Belehot) et Kapouno Henry (clan Doui), anciens élèves de l'école de Paola, qui ont connu les déplacements successifs, la première école se situait à Petit-Néami, tribu plus en contrebas de celle de Netchaot. L'éloignement et la grande dénivellation faisaient problème. C'était aussi un véritable déséquilibre pour des familles qui sont souvent du même clan mais de religions différentes.

Finalement, les vieux, après les tractations coutumières, obtinrent le déplacement, et l'école fut réinstallée à l'entrée de la tribu de Netchaot, mais à proximité du temple protestant! Le problème n'était pas résolu pour autant, la tribu étant scindée en deux zones d'influence religieuse : la zone protestante située à l'entrée de la tribu s'étire en longueur sur quelques kilomètres; la seconde, catholique, va jusqu'au fond de la tribu et jusqu'à la chefferie. Au regard des anciens le "déséquilibre" continue de se manifester : une "coutume" est exigée par les habitants de la zone catholique. Finalement après de longs palabres, les anciens décident d'un nouveau déménagement de l'école... qui est encore actuellement "posée" à la limite des deux zones d'influence et dont la cour de récréation est coupée par la petite route qui parcourt l'ensemble de la tribu! "Léquilibre" 14 étant rétabli, cette école publique ${ }^{15}$ peut devenir le point de rencontre de tous les enfants de la tribu. Sa présence introduisant ainsi un espace consensuel pour l'ensemble de la tribu.

\section{Représentation $n^{\circ} 1$}

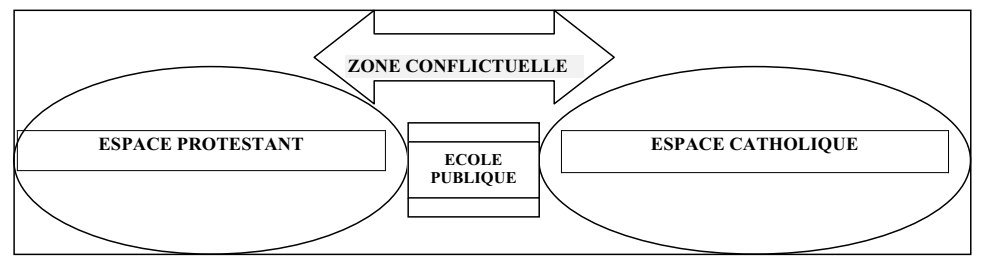

14. ClanCHÉ P. Équilibre et didactique de l'arithmétique, question de langue ou de culture? DAEST, Bordeaux, 2001.

15. Rien à voir avec une quelconque idée de laïcité. 


\subsection{L'école à la lisière de la forêt...}

L'école est très souvent construite et positionnée à l'une des deux extrémités de la tribu, soit à la lisière, soit en retrait par rapport aux habitations, à l'exemple de la maternelle de Wabao à Maré et celle de Wiwatulà Lifou. On retrouve la même configuration avec les écoles de Wakuarori, Peyec et Hnacaom. La lisière de la forêt n'est donc pas un quelconque lieu végétal (ce qui correspond dans le monde occidental à un terrain vague et en France à une quelconque ZAC). La lisière sépare deux espaces distincts, celui de la Culture ou de l'espace cultivé, Mwâciri, (Naepels, 1998 : 174) [coté tribu] et celui de l'Inculture [coté forêt] comme le dit Haudricourt (HAUdrICOURT, 1964). Que l'école y soit positionnée n'est pas indifférent...

\section{$\underline{\text { Représentation } n^{\circ} 2}$}

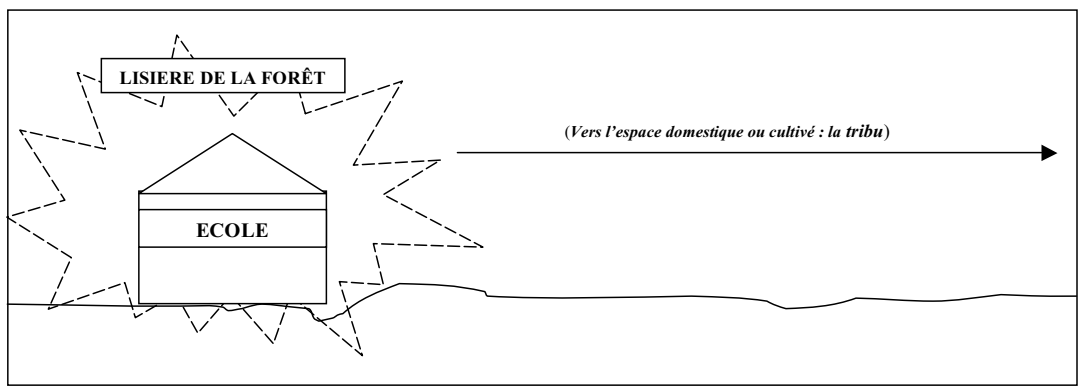

\section{...sur ou à côté du cimetière...}

Quant au cimetière, généralement implanté à la lisière ou à mi-parcours par rapport à la tribu, sa relation de proximité géographique avec l'école est manifeste dans les Îles. Ecole et cimetière partagent le même espace pour nombre de cas répertoriés. Cette disposition n'est pas inconnue pour quelques régions de la Grande Terre (on peut citer les écoles de Tye, Ko-goo-ma et Né-kwé sur la côte Est.).

Revenons aux Iles Loyauté. Tiga est l'une des plus petites îles de la Province des Iles Loyauté. La situation spatiale de son école est surprenante. L'école est sise à l'opposé de la seule tribu de l'île, à l'abri des regards même proches, un épais rideau de végétation dissimulant l'édifice ${ }^{16}$. Ce n'est pas tout : cimetière et école partagent de...".

16. Cette situation est contraire à celle de l'école de Bopope du centre Nord qui est plutôt «sous le regard 
la même cour. Seule une barrière en bois, séparation fragile, construite par les jeunes, permet d'éviter la chute des ballons sur les tombes, lors des matchs de foot improvisés. On remarquera enfin que la maison de l'infirmier occupe ce même espace d'isolement marginal. On ne peut s'empêcher devant cette contiguïté école/dispensaire de faire un rapprochement entre deux catégories de savoirs et de pratiques importés [l'école et la médecine des «Blancs»].

Cette proximité école/cimetière n'est pas exceptionnelle. Lorsqu'on a voulu agrandir la cour de récréation de l'école de Padawa, implantée sur une zone clanique conflictuelle, l'engin des travaux publics exhuma des ossements humains, à la stupeur des observateurs présents. Un "vieux ${ }^{17}$ » qui assistait à la scène révéla que la cour était partie intégrante d'anciennes sépultures, lieu du $B a o^{18}$. Certains squelettes entiers sont encore conservés dans les grottes plus en hauteur. Nous ne pouvons donc pas douter que l'école n'y soit pas positionnée intentionnellement.

L'école de Nécé, quant à elle, est située à la lisière de la forêt. Il se trouve, et ce n'est pas par hasard, que cette nouvelle parcelle attribuée soit un ancien cimetière, délocalisé depuis ${ }^{19}$. À Mébuet, tribu voisine, l'école est juxtaposée au cimetière. À Pénélo, la petite école maternelle est construite face au grand rocher Tabou du Kaze-niriz ${ }^{20}$ et proche du cimetière. À Lifou, l'école pilote du Centre-Wé (Hnasse), est à l'arrièreplan par rapport au cimetière. En Grande Terre ce phénomène est aussi très répandu (les écoles de Ko-goo-ma, de Né-Kwé, de Ouaré, de Tiaoué). Le cimetière n'est pas un quelconque lieu pour les Kanak même si cet espace a vu le jour avec la venue de la religion. Sa définition n'a strictement rien à voir avec la conception occidentale. «Lors de périples entre tribus il est conseillé aux voyageurs de se reposer près ou dans le cimetière» (BUAMA, 2003, communication personnelle). Ce n'est pas un acte irrévérencieux que d'agir de la sorte. Pour en avoir une meilleure idée, examinons succinctement les termes Bao et Kamo de la langue Ajié.

Le terme $B a o$, n'est pas aisé à définir. Etymologiquement, il suppose l'idée d'indigeste, d'a-normal, de parole répétitive, d'absence d'organisation, de nocturne et de

17. J. Guathothi, membre de la tribu du clan des Si Ecekurub de la tribu de Padawa.

18. M. Leenhartd, montre que le mot Bao est donné aux vieillards doués de sagesse ou de folie : "Moi-même, depuis longtemps missionnaire dans le pays, j'ai entendu avertir un enfant qui palpait mes souliers : Ne touche pas c'est un Bao». Haudricourt poursuit «les Bao sont les êtres de l'autre monde : dieux, mort, fantômes; ce qui correspond à la distinction européenne du naturel et du surnaturel».

19. Renseignements confirmés conjointement par Hmae Hmae (Ancien moniteur de l'école indigène de Montravel, originaire de la tribu de Nécé), Wadrawane Kawine (moniteur retraité de L’Alliance Scolaire de l'Église Évangélique) et Mme veuve Enoka Cunane dont le mari (Enoka Henri décédé) était un ancien moniteur de la vieille école de Nécé)

20. Divinité de l'Est de Maré. 
totémique des régions d'en bas. Le Bao relève du domaine du non-vivant mais intègre également l'homme doué de sagesse. Au contraire, le Kamo ${ }^{21}$ désigne le principe du vivant. Selon Leenhardt Kamo et Bao ne sont pas antithétiques. Les opposer relèverait à notre sens d'un abus de langage. Ils sont tout simplement complémentaires. Or, appliquer cette complémentarité dans le processus d'implantation de l'école, engendre singulièrement un "ailleurs" non maîtrisé, le hors-lieu où advient ce qui n’est pas produit au "centre".

Tout comme, l'église, le temple, le cimetière, l'école est posée et acceptée sur/dans un espace de conjonction. Genre d'espace de protection consensuelle où l'objet est soumis au regard et sous la protection des "vieux», Bao. Comme disait le "vieux" Mayaet, petit chef de la tribu de Werap, Salomon de Yehen (Hienhgen en Français), village de la côte Est :

«L'école, l'église, le temple tout ça c'est bon [...]. Nous, on a déjà accepté la parole. C'est bon pour les jeunes plus tard. Parce que quand, avant quand on a accepté, on ne savait pas trop, faire quoi avec cette chose. Mais on a dit, peut-être que c'est bon pour les gosses plus tard. Voilà pourquoi on a donné un bout de terrain pour l'école. Parce que aussi on a vu quand Monsieur Leenhardt est venu à Do-neva.». [Communication personnelle, 2003]

Cela est tout à fait perceptible à travers les dires de Leenhardt à propos de Cook et de ses marins "Cook raconte que les Calédoniens, devant ses marins, ne se dérangèrent pas. Ils les laissèrent circuler tandis qu' eux-mêmes demeuraient en leur place, vaquant à leurs occupations, épiant seulement les mouvements des Européens. Le lendemain ils consentirent à les guider dans les sentiers de la montagne, et ils le firent, semble$\mathrm{t}$-il, avec calme. Il y eut aussi rencontre sur le même terrain, mais sans aucun heurt, aucun incident, aucun échange réel et Cook en fut très frappé. La cause de cette réserve apparait aujourd'hui sans peine : Quand des navires aux blanches voiles venus de l'horizon, les Calédoniens virent descendre des humains astucieux et au teint clair, ils n'hésitèrent pas à voir en eux des revenants d'entre les morts de leurs familles, ceux qu'ils priaient à leurs autels rustiques, les ancêtres, les dieux incarnés...», (LEENHARDT, 1937, p. 46). On pouvait faire l'hypothèse que, tout comme les Kanak ont considéré originairement les marins comme des Bao, ils ont, plus tard, accordé à l'école un statut du même ordre. À mon sens, ce cliché issu d'une anthropologie "poussiéreuse» est aujourd'hui désuet.

21. Colombani H. Discours mythiques, symboles sacrés, in Parole, communication et symbole en Océanie. Actes de colloques C.O.R.A.I.L. et UFP, Paris, 1994, p. 67. 
Cette position spatiale manifeste objectivement la rencontre et le dialogue entre deux conceptions sociétales coprésentes. Chacun cherche à co-exister et/ou à cohabiter, quelle que soit la manière, en s'harmonisant (Attrait) et/ou en s'opposant (Expulsion/Répulsion). L'école rencontre un milieu qui n'est pas le sien et c'est le contexte qui guide son implantation. Sa position en arrière-plan du cimetière n'est pas "adversative" et la considérer comme l'expression de rejet, serait commettre un abus de langage voire un non-sens. On ne peut donc cautionner tous les discours qui relèvent de la description de deux mondes asymétriques, par extensions antagonistes. Ce genre de propos fait resurgir les interprétations archaïques voire "exotiques", sur la société. De notre point de vue, l'implantation spatiale de l'école relève d'un aspect combinatoire intentionnel. Ce positionnement n'est pas et ne peut être une donnée a-temporelle mais un produit historique élaboré à partir de contextes spécifiques convergents dans la manifestation de "l'altérité institutionnelle". En mettant en avant l'aspect combinatoire des événements et en considérant l'identité culturelle comme un processus dynamique, nous manifestons ainsi le refus de nous enfermer «dans les mondes mythico-symboliques [...] des thèses culturalistes. On ne peut alors parler de déterminisme dans la mesure où, comme insiste Bensa «rien n'est réglé par avance». (BENSA, 1998, p.56). Ne sommes-nous pas ici en présence d' «actes de langages ${ }^{22} »$ ?

\section{$\underline{\text { Représentation } n^{\circ} 3}$}

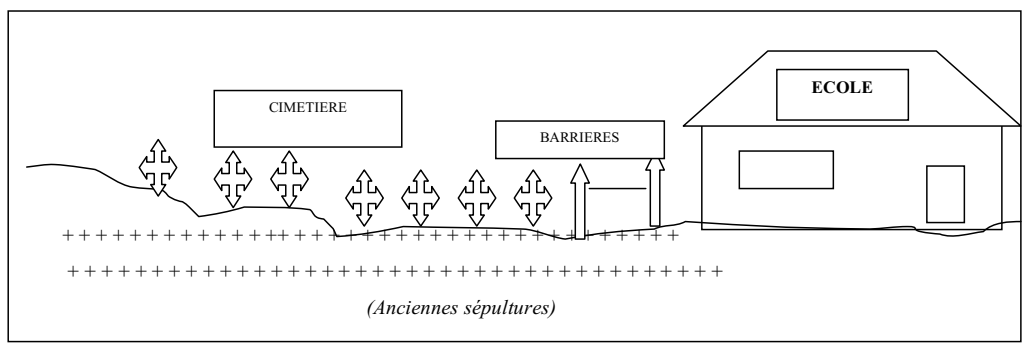

\section{L'école, attrait straté- gique : «Désir et/ou imposition d'école»}

La position cadastrale de l'école vient confirmer ce qui précède. Il suffit parfois de connaittre la nomination de certains lieux attribués à son édification pour s'en rendre compte. Si elle est "éloignée" des endroits domestiques, et "posée" à la lisière de la forêt ou sous la garde

22. Ibid., p. 129. 
des Atressi, c'est qu'on lui accorde le statut d'objet de valeur engendrant prestige, pouvoir et richesse. Ceci vient en contradiction avec une forme de pensée occidentale présidant à l'organisation territoriale de l'administration coloniale pour laquelle tout ce qui est prestigieux doit demeurer au "centre", et à la "périphérie" ce qui est éphémère ${ }^{23}$.

"Quand j'ai grandi, l'ancienne école est au bout de la tribu, juste avant l'entrée de la tribu voisine, c'est-à-dire Traput. Donc l'école était complètement décentrée par rapport à la tribu. Ce qui n'est pas le cas de l'école d'aujourd'hui. Â l'ancienne école il y avait juste une maison, c'était la famille Ukeiwe. Comme je disais tout à l'heure, l'école a été construite au bout de la tribu dans Jozip près de celle de Traput mais sur le terrain de la grande chefferie de Lössi d'où la présence de la famille Ukeiwe. Et si les gens de Traput revendiquent l'école on dira qu'elle appartient à la chefferie de Lössi. C'est aussi une stratégie pour la récupérer après en disant, après tout le grand chef a donné à l'école publique. Donc maintenant je peux la réutiliser.» [Kahtanye, A. 2000. Communication personnelle].

Considérons le cas d'une école de tribu de Lifou construite entre les années 1948 et 1950. À cette époque, la plupart des enfants de l'île fréquentaient les écoles missionnaires. Ces structures drainaient l'ensemble des enfants en âge scolaire de l'île. Néanmoins la grande distance qui les séparait des tribus dissuadait bon nombre de familles d'envoyer leurs enfants dans ces établissements. Pour la tribu citée ci-dessus cette question de distance était problématique. La généralisation ainsi que le prestige de l'école publique sur l'île, faisaient que :

«Les gens commençaient à reconnaitre que c'est un outil pour l'instruction. [...] Les parents voyaient qu'avec l'école publique on pouvait aller plus loin. Les écoles confessionnelles de cette époque n’avaient que [...] deux objectifs : l'éducation religieuse et l'éducation à la vie» [Kahtanye, 2000].

Obtenir une école publique de proximité devenait nécessaire. L'administration est alors interpellée. On procède aux négociations nécessaires à l'implantation d'une école et les instances gouvernementales engagent le dialogue avec les autorités coutumières en privilégiant les tractations avec le petit chef de la première tribu que nous appellerons désormais $\mathbf{J}$, personnage qui semblait être à même de peser dans les décisions

23. cf. le décret du 26 septembre 1902, article 1, à propos de la réglementation et de l'organisation de l'école publique en Nouvelle-Calédonie. 
futures. Dorénavant et dans des circonstances inaccoutumées, J se trouve placé "sous le regard de"... et "exposé à"... Pour se soustraire à cette incongruité culturelle tout en manifestant le désir de voir cet objet riche placé dans sa zone d'influence, J entre à son tour en discussion avec le clan Atressi ou Acania habitant à la lisière, zone frontalière entre les deux tribus. Un autre accord tacite est convenu avec un second petit chef (désormais T), celui de la tribu voisine. Une fois la décision arrêtée, les autorités coutumières proposent un endroit excentré, une portion de terre contrôlée par les Atressi. Donc, une seule école est construite à la limite, capable d'accueillir les enfants des deux tribus.

À première vue tout paraît serein et équilibré. Cependant les choses ne sont pas si simples. Puisqu'en posant cet objet dans cet espace, $\mathbf{J}$ se trouve impliqué dans les enjeux sociaux contextuels : i) Le choix de l'administration de privilégier un seul acteur lors des négociations en l'occurrence J lui-même; ii) L'attribution d'un espace contrôlé par le clan Atressi plus ou moins le représentant par alliance de la grande chefferie de tutelle de la localité; iii) $\mathrm{T}$ et les parents de la tribu contiguë; or T n'est pas affilié coutumièrement au même district que J... Recherche d'emprise et lutte d'influence s'annoncent. Le déplacement de l'école semble inéluctable...

Quelques années s'écoulent avant que les événements prennent une autre orientation pour J. Rappelons-nous que si l'administration l'avait choisi quelques années auparavant comme interlocuteur privilégié, c'est qu'il avait déjà un engagement politique ${ }^{24}$. Devenu un élu de l'Assemblée Territoriale ${ }^{25}$ par voie électorale, son pouvoir décisionnel est renforcé voire accru et l'histoire de cette petite école va rebondir.

L'augmentation des effectifs scolaires appelle l'agrandissement de l'école. Comme J est le seul représentant de l'administration dans la tribu, il est logique qu'il s'octroie le droit d'être le principal dépositaire de ce bien "administratif» prestigieux. Une fois toutes les conditions réunies et sous le couvert du clan Atressi sa première décision est de déplacer ce bâtiment de la zone frontalière pour le mettre au centre de "sa" tribu à proximité de ses habitations, entre les années 1960 et 1970 d'après notre interlocuteur :

"La nouvelle [école], est devenue l'école de sa tribu, dorénavant on dira c'est son école et il œuvre pour le bien de ses frères ${ }^{26}$ ».

24. C'était l'époque de la Loi Cadre (1956) dite loi Deferre, qui entendait «faire participer directement toutes les ethnies, et en particulier les Mélanésiens, à la gestion des affaires publiques» (BENSA, 1998, p. 108).

25. Actuellement le Congrès de la Nouvelle-Calédonie.

26. Nous préférons le terme "frères» car il est plus approprié que le terme «sujet». Le chef a des frères (lignage) et non des sujets. Le terme «sujet» est un héritage de l'histoire qui engendre des ambiguïtés dans le milieu Kanak. 
Ce qui est indubitablement légitime puisqu'il agit en tant qu’ainé et représentant de l'administration. C'est ce que confirme notre interlocuteur lorsqu'il déclare :

«Le chef de ma tribu est très rodé politiquement, à mon avis il avait déjà une idée sur l'importance de l'école et de l'importance du rôle qu'elle peut jouer. Notamment par la mise en place de cette école publique, comment il peut favoriser la promotion de ses sujets». [Kahtanye, 2000].

Dans le milieu kanak espace et pouvoir sont intimement liés et on ne déplace pas impunément sur une simple décision un objet "posé" dans un espace. Le pouvoir est le résultat d'un consentement mutuel. On ne devient pas grand chef ou petit chef par droit divin. C'est le contrat qui permet d'asseoir une certaine légitimité au pouvoir. Le chef n'est pas couronné en tant que monarque mais "installé" au sens d'“arrangement" pour être "accrédité" du pouvoir qui lui est octroyé.

Examinons ce processus de déplacement qui contredit en apparence la théorie de l'implantation à la lisière. Le rôle des Atressi est ici conséquent. Ils sont là pour garantir une certaine harmonie entre les différentes instances tribales. L'Atressi est considéré comme :

«[...] un verrou filtrant. Il peut fermer comme il peut autoriser. [...] Ce n'est pas un verrou qui condamne définitivement. Il permet la souplesse du système.» [WELEPA, 2000. Communication personnelle].

Ils sont aussi comme le dit $\mathrm{A}$. Buama : «Le filtre ou le sas par où transite tout objet quel qu'il soit, tous discours et toutes décisions concernant les rouages ou les tractations coutumières impliquant la grande chefferie». (BUAMA, 2003)

J ne peut ignorer les Atressi, qui sont à la fois placés sous sa juridiction et aussi sous celle de la grande chefferie située à quelques kilomètres de sa propre tribu. Rappelons que la fonction des Atressi est de rechercher tant bien que mal, les moyens d'établir les conditions nécessaires aux rééquilibrages sociopolitiques internes. Pour J., s’allier aux Atressi représente une disposition échappatoire symbolique à l'emprise de la grande chefferie. Ce clan i) constitue une force de tampon en cas de conflit; ii) représente une force d'équilibre “dynamique”. C'est l'une des raisons de la présence de l'école sur leur terre, zone frontalière; iii) "C'est aussi un enjeu personnel par rapport à $\mathrm{T}$ ». [KAHTANYE, 2000].

Ainsi, placer l'école sur le terrain des Atressi, c'est tenter de protéger l'objet des convoitises internes et chercher à le "neutraliser", en vue de lui attribuer un nouveau statut, d'où notre expression : «Accueil stratégique». En cas de revendication, «on dira que c'est l'école de la grande chefferie, $[. .$.$] ».$ 
En acceptant cette disposition - école en zone frontalière - $\mathrm{J}$ gagne la confiance des Atressi. Car J rend dynamique une des fonctions culturelles du clan cité précédemment : renforcer implicitement les assises politiques, économiques et socioculturelles du coopérateur. Par ce procédé il ratifie les anciennes alliances et réaffirme cet espace symbolique intermédiaire qui l'a toujours soutenu et protégé, ce qui le conduit à se positionner en force de pouvoir face à $T$. L'assise acquise auprès de l'administration conforte ses éventualités décisionnelles. Stratégiquement, fédérer les Atressi et l'administration à son projet c'est asseoir son pouvoir, son prestige, la promotion de ses sujets, [et] sa position vis-à-vis de la tribu appartenant au district adverse.

Les actions menées par $\mathrm{J}$ servent en fait à re-légitimer implicitement son statut d'aîné. Dans le temps, son ancêtre qui était un des aînés de la grande chefferie, avait été écarté de peur de fomenter une rébellion et contester le pouvoir en place. On ne le tua pas alors par crainte, car il appartenait à la lignée des chefs. Il fut simplement déplacé et gardé en lieu sûr, en l'occurrence dans cette tribu sous la «présence ${ }^{27}$ » et la responsabilité du clan Atressi. Lui et toute sa descendance peut grandir, en marge et attendre en quelque sorte la venue de l'école pour pouvoir récupérer autrement ce qui était dû. Et Kahtanye d'ajouter : «C'était le moment idéal pour régler ses différends avec la grande chefferie [...]». L'«école» devient une opportunité pour retrouver un pouvoir perdu. Elle est donc "détournée, déviée» - aussi - de sa fonction première pour être instrumentalisée dans une perspective stratégique et personnelle. Cela montre aussi la plasticité des sociétés traditionnelles capables de "phagocyter" et de transmuter tout ce qui est exogène. Qu'en est-il du savoir de l'école?

\subsection{Stratégie et Ruse}

L'école, à son insu, tonifie deux dimensions fortement présentes dans la culture, à savoir "la fonction de pouvoir" [symbolique] et de "prestige". Nous discernons ponctuellement l'opportunité de la définition du «contexte» chez Naepels et de la pertinence de l'expression "contexte entrelacé» chez Bensa. J est dirigeant coutumier, politicien, émissaire de l'administration dans la tribu et homme d'église. Il développe son action sous ce que l'on nomme maintenant une identité plurielle. En suivant au plus près le fil de cette action, on voit bien comme l'affirment les ethnométhodologues, que le social se construit localement et continuellement. À l'intérieur de cadres au sens Goffmanien, les acteurs agissent alors en fonction d'un «vouloir faire ou d'un vouloir

27. Le terme "présence» ne renvoie pas à celui de surveiller. Il évoque une «cohabitation» ou le «vivre avec» avec une connotation proxémique. 
dire» (QUERÉ, 1990 : 84). La procédure du positionnement puis du déplacement de l'école est la résultante d'une spéculation ou l'on entrevoit une sorte de «ruse». Celleci n'est pas sans évoquer ce dont parlent Détienne et Vernant (2002), feindre d'obéir pour éviter trop de brutalité dans l'oppression!

\subsection{Une extranéité sans ostracisme}

L'école est donc précipitée dans un milieu culturel où des rapports sociaux spécifiques assignent des modalités formelles d'occupation de l'espace. La localisation marginale en apparence de l'école en est la traduction. Manifestement, elle est "posée" quelque part, dans un espace bien défini, en l'occurrence ici [sa proximité avec] le cimetière. Si elle occupe cette position, c'est qu'elle représente autre chose que ce qu'elle est réellement; son statut est donc spécifiquement construit. Tout comme la position de l'étranger, Bao, dans le milieu, l'infrastructure scolaire n'est "ni rejetée, ni intégrée" mais "placée" dans la position qui convient au pouvoir qui lui est accordé, "juste là où il faut"... Le milieu compose avec l'étranger, offrant à l'observateur une œuvre culturelle. Exprimée à travers les concepts bilatéraux de réciprocité : «Bao-Kamo»et de «Culture-Inculture». Il n'y a donc pas d'opposition entre ces deux entités spatiales, elles sont uniquement distinctes selon leur spécificité. Exprimer cette extranéité en terme d'exclusion est donc illégitime et non advenu voire contraire à la pensée kanak. On comprend pourquoi Haudricourt, affinant la dichotomie radicale levistraussienne Nature vs Culture, propose une suture ambivalente entre "Culture" et "Inculture" (HAUDRICOUT, 1964 : 102). La co-habitation école-cimetière est donc intentionnellement culturelle. Cet «ailleurs» est intégré dans un genre de «sacré local, non-dit». Qui, à notre sens, n'est cependant perceptible qu’à travers «une interlocution singulière» (BENSA, 1999). À défaut, pratiquer une forme «d'immersion longue chez les vaincus, pour vivre de l'intérieur leur vision $[\ldots]^{28}$ ». Car l'espace géographique ne nous dit rien.

La capacité de l'habitant à ménager ou manipuler l'objet entre le "connu" et l'“inconnu" relève d'une construction "indigène”. Le résultat en est cette école logiquement et paradoxalement positionnée.

28. ClanCHÉ P. Parler de ceux qui vous ont adopté? Expérience et réflexions sur la relation de familiarité en anthropologie de l'éducation. In : Les Sciences de l'éducation - Pour l'ère nouvelle, vol. 38, n 1, 2005, pp. 11-41. 
Les Sciences de l'éducation - Pour l'Ère nouvelle, vol. 41, nº 1, 2008

\subsection{L'art de l'arrangement}

L'école est donc au croisement d'un entrelacs de contextes. Un lieu propre lui est assigné, ambigu et médiateur, nécessaire et redoutable. Nombreuses sont les écoles de tribu dont l'implantation spatiale répond plus à une recherche de cohésion ou de résolution d'un problème local qu'à un véritable rapport avec les enjeux de la scolarisation. Comme dit Welepa :

«L'école est pour ainsi dire, manipulée comme un objet [...] de valeur et parfois déviée de son véritable rôle institutionnel». [Communication personnelle, 2002].

Elie Poigoune, premier professeur kanak certifié de mathématiques au Lycée La Pérouse et longtemps très engagé dans la lutte indépendantiste, ne déclarait-il pas dernièrement :

«[...] je pense par rapport à l'école, $[\ldots]$ c'est bien la chose qui a été la moins coloniale [...]!». [Communication personnelle, 2002].

On n'en est pas à un apparent paradoxe près!

La position spatiale d'école est alors à prendre au sens des «logiques métisses» dont parle J-L. Amselle (1999), et non au sens d'une hypothétique expression mythique à la Levi-Strauss (BENSA, 2003, p. 49), car l'école comme élément historique intègre un autre contexte où Culture et Histoire - ici l'histoire coloniale — se combinent singulièrement (SAHLIN, 1989). Comme le dit Amselle "toute culture gère sa perméabilité, toute culture inscrit ses extériorisations locales dans un contexte microhistorique et politique qui en modifie les manifestations». Considérer les tiraillements entre les autorités insulaires pour avoir l'école et qui a pour conséquence leurs déplacements successifs puis leurs positionnements sur des lieux ambigus, démontre la pertinence de les analyser à travers "une anthropologie historisée et généralisée ${ }^{29}$ ".

Tout ceci prouve aussi qu'il n'y a pas de règle formelle ou formalisée : "Qui dit que...! Qu'il faut que...!». Tout "est" ou "dans" ou encore "dépend" de la situation souvent spontanée du «jeu» social dans lequel les règles sans cesse rediscutées redéfinies ne sont pas forcément apprises et connues.

Qu'en est-il de l'école avec ses règles formelles lorsqu'elle est confrontée à de telles situations?

Aujourd'hui en Nouvelle-Calédonie l'enseignant ne peut plus être un simple répétiteur..., car l'élève a besoin de repérer le niveau d'exigence des compétences exercées.

29. Op.cit., p. 136. 


\section{Conclusion}

Notre but n'est ni de faire de l'ethnocentrisme à rebours ni d'opposer les terminologies «eux/nous», clivage de logique sociale de la culture du pauvre (HOGGART : 1970). Nous voulions simplement exposer et comprendre la pratique kanak vis-à-vis d'un objet - École - surgissant dans un milieu déjà saturé de culture et d'histoire. L'analyse des arrière-plans culturels dans ce processus de localisation ne relève pas de la simple curiosité exotique. Foncièrement complexe, cette situation spatiale paradoxale se révèle un analyseur de la place que les notables kanak ont assigné aux savoirs de l'école de blancs : objets non pas nécessaires (du moins dans les débuts) ni réellement contraints, mais objets à la fois attirants - du seul fait de leur nouveauté -, et dangereux - du fait de la menace qu'ils font peser sur les équilibres coutumiers toujours instables -. Ce rapport aux savoirs occidentaux et à leur mode de diffusion pèse encore fortement un siècle après. Il conviendrait peut-être, rompant avec un exotisme détestable et sans tomber dans un historicisme désuet, de comparer l'implantation désaxée des premières écoles de brousse et des îles avec celle, centrée, des institutions Jules Ferry où école des garçons et école des filles constituaient les ailes du bâtiment de la mairie, faisant face à l'église comme par défi. Certes le contexte historico-politique était bien différent. Mais il serait sans doute révélateur de dépasser les images d'Épinal de la préparation de la revanche de Sedan, et d'interroger de près le milieu local d'alors : on y trouverait peut-être, certes sous une forme moins spatialisée, des enjeux comparables à ceux que nous avons modestement recueillis sur la Grande Terre et dans les Iles.

En tout cas, il ne sert à rien de gémir sur l'échec des élèves kanak si l'on ne prend pas le temps de regarder comment ces savoirs occidentaux sont perçus et mis à leur place par les acteurs du système. 
Les Sciences de l'éducation - Pour l'Ère nouvelle, vol. 41, $\mathrm{n}^{\circ}$ 1, 2008

\section{Bibliographie}

AMSelle J. L. Logiques métisses, Anthropologies de l'identité en Afrique et ailleurs. Paris : Payot, 1999.

BAZIN J. Questions de sens. Enquête, 1998, nº 6.

BeNSA A. De la micro-histoire vers une anthropologie critique. In : REvel J. Jeux d'échelles, La micro-analyse à l'expérience. Paris : Gallimard/Le Seuil, 1996.

Bensa A. Nouvelle-Calédonie, vers l'émancipation. Paris : Gallimard, 1998.

BENSA A. À quoi sert la notion de culture? Actes du XIII colloque CORAIL, Nouméa, 2003.

Bensa A. La fin de l'Exotisme, Essai d'anthropologie critique. Toulouse : Anacharsis, 2006.

Bensa A. \& Freyss J. La société kanak est-elle soluble dans l'argent...? Terrain, 1994, $\mathrm{n}^{\circ}$ 23. Mis en ligne le 21 juillet 2005. URL : http://terrain.revues.org/ document3098.html, consulté le 16 juillet 2006.

Bourdieu P. \& Passeron J-C. les héritiers. Paris : Minuit, 1964.

BOURDiEu P. La misère du monde. Paris : Seuil, 1993.

BRUNER J. Car la culture donne forme à l'esprit; de la révolution cognitive à la psychologie culturelle. Paris : Eshel, 1991.

BRUNER J. L'Éducation, entrée dans la culture : les problèmes de l'école à la lumière de la psychologie culturelle. Paris : Retz, 1996.

ClanchÉ P. L'enfant et le contrat didactique dans les derniers textes de Wittgenstein.

In : Hannoun H. \& Drouin A-M. Pour une philosophie de l'éducation. Dijon : CNDP/CRDP de Bourgogne, 1994, pp. 223-232.

Clanché P. Le problème de la prise en compte des ressources culturelles locales d'apprentissage dans une école privée kanak. In : FEGER R. (Ed.). L'éducation face aux nouveaux défis. Montréal : Editions Nouvelles, 1996, pp. 274-278.

Clanché P. The Didactic and Coutumier Contracts; a contribution to the issue of cultural backgrounds in formal education in New Caledonia. In : PEDDIE R.A. Looking at the past, looking to the future : educational change in comparative perspective, Auckland, 1998, pp. 40-50. 
Clanché P. Un aspect du métier d'élève chez le jeune enfant Kanak : écouter, comprendre, faire, écrire. Revue française de pédagogie, 1999, nº 127, pp. 99-106.

ClanCHÉ P. Anthropologie de l'éducation et didactique des mathématiques : pour une anthropo-didactique. In : Didactique des discipline et formation des enseignants : approche anthropologique, Marseille, 2000. http://pagesperso-orange.fr/daest/ Textes\%20en\%20ligne/Clanche_Marseille2000.pdf, consulté le 29.02.2008.

Clanché P. Histoire d'implantation et d'investissement des lieux d'instruction, et représentation de la transmission des savoirs. In : Peyronie H. \& Vergnioux A. (dir.). Éducation et longue durée. Caen : PUC, 2007, p. 107.

Coulon A. Ethnométhodologie et éducation. Paris : PUF, 1993.

Coulon A. L'école de Chicago. Paris : PUF, 1994.

DARDELIN M-J. L'avenir et le destin, regards sur l'école occidentale dans la société kanak. Paris : ORSTOM, 1984.

Detienne M. \& Vernant J-P. Les ruses de lintelligence; la mètis des Grecs. Paris : Flammarion, 2002.

SHINEBERG D. Ils étaient venus chercher du santal. Bulletin de la Société des études historiques de la Nouvelle Calédonie, 1981, $\mathrm{n}^{\circ} 3$.

Doumenge J. P. Du terroir... à la ville. Travaux et documents de géographie tropicale, $1982, n^{\circ} 46$.

GAUTHIER J. Les écoles populaires Kanak, une révolution pédagogique? Paris : L'Harmattan, 1996.

HaUdricourt G. Nature et culture dans la civilisation de l'igname : Origine des clones et des clans. L'Homme, 1964, vol. 4, n 1, pp. 93-104.

Hoggart R. La culture du pauvre. Paris : Minuit, 1970.

FouCAult M. Surveiller et punir. Paris : Gallimard, 1975.

Goffman E. Les cadres de l'expérience. Paris : Minuit, 1991.

ILlOuZ C. De chair et de pierre : essai de mythologie kanak. Paris : MSH, 2000.

KOHLER J.-M. \& WACQUANT L. L'école inégale, Éléments pour une sociologie de l'école en Nouvelle Calédonie. Nouméa : ORSTOM, 1985.

LAmbert J.-M. La Nouvelle politique indigène en Nouvelle-Calédonie : le capitaine Meunier et ses gendarmes 1918-1954. Paris : L'Harmattan, 1999. 
Les Sciences de l'éducation - Pour l'Ère nouvelle, vol. 41, n 1, 2008

Levi-Strauss C. La pensée sauvage. Paris : Plon, 1962.

LEENHARDT M. Gens de la grande terre. Paris : Gallimard, 1937.

LeEnHardT M. Do kamo. Paris : Gallimard, 1947.

NaEPels M. Histoires de terres Kanakes (Conflits fonciers et rapports sociaux dans la région de Houaïlou. Nouvelle-Calédonie). Paris : Belin, 1998.

Pineau-Salaün M. Les Kanak et l'école, Socio-histoire de la scolarisation des Mélanésiens de Nouvelle-Calédonie (1853 - 1998). Thèse de Doctorat en sociologie (préparée sous la direction de Monsieur Christian Baudelot). Paris : EHESS, 1999, 889 p.

Pineau-Salaün M. L'école indigène, Nouvelle-Calédonie, 1885-1945. Rennes : Presses Universitaires de Rennes, 2005.

Queré L. Agir dans l'espace public. In : Queré L. Les formes de l'action. Paris : EHESS, 1990, $343 \mathrm{p}$.

SAHLIns M. Des îles dans l'histoire. Paris : Gallimard/Le Seuil, 1989.

SAnd C., Bole J. \& Ouetcho A. Les sociétés préeuropéennes de la NouvelleCalédonie et leur transformation historique : l'apport de l'archéologie. In : BENSA A. \& Leblic I. (dir.). En pays Kanak. Paris : Éditions de la maison des Sciences de l'Homme, 2000.

SARRAZY B. La sensibilité au contrat didactique. Rôle des arrière-plans dans la résolution de problèmes d'arithmétique au cycle trois. Thèse de Doctorat en Sciences de l'éducation. Bordeaux : Université Victor Segalen Bordeaux 2, 1996.

WaCHTEL N. La vision des vaincus : les Indiens du Pérou devant la conquête espagnole 1530-1570. Paris : Gallimard, 1971.

WadraWANE E. Situation spatiale paradoxale de l'école en milieu kanak. DEA en Sciences de l'éducation. Bordeaux : Université Victor Segalen Bordeaux 2, 2001.

WadraWane E. Métissage et Culture du métissage en Nouvelle-Calédonie (Groupe de Recherche en Histoire Océanienne Contemporaine). Paris : Les Indes Savantes, 2003.

Wittgenstein L. De la certitude. Paris : Gallimard, 1965.

Woods P. L'ethnographie de l'école. Paris : Colin, 1990.

Wolton D. L'autre mondialisation. Paris : Flammarion, 2003, pp. 49-50. 


\section{Positioning and moving Kanak-medium schools \\ An anthropological analysis of the place of institutions diffusing western knowledge in a colonial context}

Abstract: In New Caledonia and in predominantly Kanak tribal areas, schools are generally located far from the social centre of the group. Outside that social / domestic sphere, a peripheral ring is devoted to those same schools. Even though they happen to be oddly placed outside the tribal areas, schools are neither fully integrated nor completely rejected but located just where they should be, according to the authority they are allocated. The history of such location and the current situation is a good indication of the importance Melanesian people give to those institutions dispensing with western knowledge and of the importance they give to such knowledge.

Key words: Schools in indigenous communities. Kanak Communities. Knowledge Development. New Caledonia. Province of the Loyalty Isles.

\section{Localización y desplazamiento de las escuelas en el entorno Kanak}

Un analizador antropológico del lugar dedicado a las instituciones de difusión del saber occidental en una situación colonial

Resumen : En Nueva Caledonia y en el entorno Kanak, es decir, en lo que se llama "las tribus ", la escuela está por lo general implantada lejos del espacio en el que se concentra la vida social del grupo. Fuera de este espacio social/doméstico se le reserva una banda periférica. Aunque esté particularmente descentrada la escuela no está ni integrada ni rechazada sino situada en la posición que conviene al poder, justo donde debe ser. La historia y la actualidad de este modo de implantación constituyen un indicador del lugar concedido por los melanesianos a las instituciones de difusión del saber occidental y al lugar que ocupa este mismo saber.

Palabras claves : Escuela indígena. Espacio Kanak. Relación al saber. Nueva Caledonia. Provincia de las Islas Lealtad.

Eddy Wayuone WadraWANE. Emplacement et déplacement des écoles en milieu Kanak. Un analyseur anthropologique de la place faite aux institutions de diffusion du savoir occidental dans une situation coloniale. Les Sciences de l'éducation - Pour l'Ère nouvelle, Apprentissage des mathématiques et élèves «différents», vol. 41, n 1, 2008, pp. 115-139. ISSN 0755-9593. ISBN 978-2-9528025-5-0. 